\title{
Behavioral inhibition induced by ingestion in sea anemones (Anthopleura elegantissima)
}

\author{
CHERYL A. LOGAN \\ University of North Carolina at Greensboro, Greensboro, North Carolina 27412
}

\begin{abstract}
Following tests for initial responsiveness, the suppressive effect of ingestion of Artemia salina on the contraction response of the sea anemone, Anthopleura elegantissima, was assessed. During testing, repeated presentations of a water-stream stimulus occurred over a period of $70 \mathrm{~min}$. All subjects also underwent control testing in which the water-stream sequence was presented in the absence of prior Artemia ingestion. Significant suppression of oral disk contraction to the water stream was observed following Artemia ingestion with and without water exchange during testing. The relationship of this finding to similar results obtained with Hydra is discussed.
\end{abstract}

Rushforth $(1967,1971,1973)$ has demonstrated the occurence of two forms of behavioral inhibition among several species of coelenterates of the class Hydrozoa. Response decrements similar to behavioral habituation as described in many vertebrates can be produced in Hydra by repetitive mechanical stimulation. In addition, however, a more immediate decline in contractions of the body column may follow the single presentation of a selected stimulus. Five minute exposure either to illumination or to ingestive substances such as live Artemia salina (brine shrimp), extract of Artemia, or reduced glutathione (GSH) produces immediate cessation of responsiveness to mechanical agitation (Rushforth, Krone, \& Brown, 1964). Unlike the case with habituation, these effects require no repetitive stimulation. Following a single inhibitory stimulus, however, the successive presentation of mechanical stimulation at 1-min intervals reveals a resumption of responding after from 30 to $70 \mathrm{~min}$.

Several reports suggest that similarly distinct types of behavioral inhibition may characterize Anthozoans as well. Habituatory decrements in oral disk contraction to mechanical stimulation are well known in Anthozoans (Logan, 1975; Logan \& Beck, 1978), and early reports are suggestive of a more immediate form of behavioral inhibition as well. Jennings (1905), for example, reported that well-fed Aiptasia are much less responsive to mechanical shock than are deprived animals. Similarly, water disturbances that elicit strong reactions in hungry sea anemones have little effect on sated individuals. That

This research was supported by Grant 20752-01 from the National Institutes of Health, Edmund Fantino, Principal Investigator, and by the Alfred P. Sloan Foundation. Requests for reprints should be sent to Cheryl A. Logan, Department of Psychology, University of North Carolina at Greensboro, Greensboro, North Carolina 27412. these reports may parallel the glutathione-induced inhibition of contraction described for Hydra is further suggested by the similarity of the chemical control of feeding in many species of Hydrozoa and Anthozoa. Reduced glutathione (GSH), known to activate complex feeding reactions in Hydra (Lenhoff, 1968; Loomis, 1955), also elicits the ingestion phase of feeding in the Anthozoan Anthopleura elegantissima (Lindstedt, 1971). A complete feeding reaction in Anthopleura results from presentation of either live Artemia salina or extract of Artemia tissue fluids. Immediately following Artemia presentation, the anemone's tentacles contract and bend toward the mouth, which begins to swell and open before contact is made with the food. Following contact, the Artemia is transferred to the mouth and ingested. Only ingestion, however, is elicited by GSH. Earlier phases of the feeding response (the contraction and bending of the tentacles, and the transfer of food to the mouth) in Anthopleura are elicited by asparagine.

These observations suggest that immediate decrements in responsiveness to mechanical stimulation similar to those described in Hydra may be produced in Anthopleura as well. Under normal conditions, the presentation of a single moderateintensity stream of water evokes a vigorous contraction of the animal's oral disk. With repeated stimulation, this response exhibits behavioral habituation. The present experiment tests the effects of the single presentation of live Artemia salina on the anemone's responsiveness to water-stream stimulation.

\section{METHOD}

\section{Subjects and Apparatus}

Eight Anthopleura elegantissima collected from the intertidal regions on the beaches of San Diego, California, were employed 
as subjects. Of these, seven had undergone previous testing in a habituation paradigm. One subject (S5) died suddenly in the middle of the experiment. Animals were individually housed in $500-\mathrm{ml}$ Pyrex beakers, and each underwent daily water changes of $300 \mathrm{ml}$ of filtered seawater maintained at a temperature of $21^{\circ} \pm 1^{\circ} \mathrm{C}$. Mechanical stimulation was provided by a constant-intensity stream of fresh water presented through a Water Pik oral irrigating appliance, the hose and handle of which were suspended directly above the organism, $22 \mathrm{~cm}$ from the bottom of the beaker. Duration of the stimulus and interstimulus interval was programmed on LVE multifunction timers. A timer operation activated the Water Pik jet for a controlled period by continuous input to an ac relay connected to the Water Pik. The ingestive stimulus consisted of the presentation of 10 to 15 live Artemia salina. Subjects were fed only during testing, and laboratory lighting conditions followed a continuous $12 / 12$-h period of light/dark alternation. Response measurement was accomplished photographically using a Bauer C1M Super 8 movie camera equipped with a single-frame cable release, and programmed to advance the film one frame at a time in synchrony with the presentation of the water-stream stimulus. In this way, continuous photographic records of changes in the tentacle-to-tentacle diameter of the animal's oral disk were obtained.

\section{Procedure}

Following a single pretest trial to assess the subjects' initial responsiveness to the water stream, each animal received one Artemia trial during which from 10 to 15 live Artemia were placed simultaneously in the beaker. Duration of the Artemia trial ranged from 15 to $35 \mathrm{~min}$, depending on the duration of the feedings response. After this time any noningested Artemia were removed by suction. The subjects then underwent a series of 70 test trials, each consisting of the presentation of a .05-sec-duration stream of fresh water. The interval between trials was fixed at $1 \mathrm{~min}$. Results from pilot subjects tested with shorter Artemia trials of duration approximately equal to $15 \mathrm{~min}$ indicated that the longer interval is required to insure completion of the entire feeding response. Animals that are tested too quickly following contraction and reopening respond by immediately contracting and then regurgitating all ingested materials.

All subjects were tested under conditions in which no water exchange occurred during the 70-trial test sequence. One-half of these animals (cf. Table 1) were subsequently retested under conditions in which the virtually complete exchange of seawater gradually transpired during testing. Water exchange was accomplished using an open hose siphoning system in which fresh seawater continually drained into the beaker as the beaker seawater was continually siphoned out. Under this condition, therefore, both the salinity of the seawater in the beaker and the beaker water level remained constant throughout the session.

Each of the eight subjects also underwent control testing in which 70 water-stream trials were presented, preceded, however, by no Artemia trial. For half of the subjects $(4,5,6$, and 8 ), the control sequence occurred under conditions of no water exchange, and, with one exception, these subjects underwent control testing a minimum of 4 days after the Artemia test. For the four subjects $(1,2,7$, and 9$)$ tested under both no exchange and water exchange, controls were conducted with and without water exchange. Controls with water exchange occurred a minimum of 4 days prior to Artemia testing.

\section{Results}

Artemia presentation elicited immediate and vigorous feeding reactions in all of the subjects tested. Moreover, Artemia ingestion produced a pronounced inhibitory effect on subsequent responding to the water stream. The inhibitory effect was in evi-
Table 1

Contraction Responding Before and After Artemia Ingestion Calculated as a Percentage of the Subject's Resting Diameter

\begin{tabular}{cccccc}
\hline & \multicolumn{2}{c}{ No Exchange } & & \multicolumn{2}{c}{ Exchange } \\
\cline { 2 - 3 } \cline { 5 - 6 } Subject & $\begin{array}{c}\text { Pre- } \\
\text { Artemia }\end{array}$ & $\begin{array}{c}\text { Post- } \\
\text { Artemia }\end{array}$ & $\begin{array}{c}\text { Pre- } \\
\text { Artemia }\end{array}$ & $\begin{array}{c}\text { Post- } \\
\text { Artemia }\end{array}$ \\
\hline 2 & 35.7 & 54.7 & 22.2 & 71.6 \\
3 & 23.2 & 69.1 & 35.7 & 46.4 \\
4 & 54.3 & 62.9 & & \\
5 & 47.2 & 52.8 & & \\
6 & 50.0 & 81.3 & & \\
7 & 45.5 & 39.4 & 25.8 & 71.9 \\
8 & 12.8 & 64.1 & & \\
9 & 28.9 & 91.2 & 25.6 & 46.2 \\
Mean & 37.2 & 64.44 & 27.33 & 59.02 \\
\hline
\end{tabular}

dence both with and without the exchange of seawater in the test beaker. The most representative outcome was a partial suppression of oral disk contractions on the initial water-stream trials, followed later by more complete suppression and, occasionally, recovery. Table 1 compares the mean tentacle-to-tentacle diameter on the first three-trial block following Artemia ingestion with that measured on a single water-stream trial just prior to ingestion. Both are calculated as a percentage of the animals's resting diameter, therefore, the smaller the percentage, the greater the contraction response. Inspection of the group means reveals significantly less responding following ingestion, either with or without water exchange [no exchange: $t(7)=3.5, p<.01$; exchange: $\mathrm{t}(3)=4.2, \mathrm{p}<.025]$.

In one animal, the difference between pre- and post-Artemia responsiveness was as great as $60 \%$ of the resting diameter (Subject 9). Figure 1 illustrates the virtually complete inhibition of responsiveness to mechanical stimulation in Subject 9. While, as indicated by the asterisk, substantial responding oc-

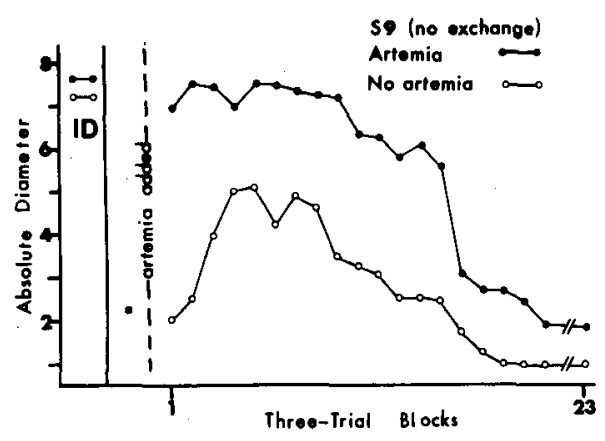

Figure 1. Three-trial block means of the subject's responding to water-stream stimulation both following and in the absence of prior ingestion of live Artemia salina. The data represented were collected under conditions of no exchange of the nuid medium. ${ }^{*}=$ responsiveness to water-stream prior to ingestion; ID = initial resting diameter.) 
curred prior to Artemia ingestion, the presentation of the water stream following ingestion resulted in no oral disk contraction. In the absence of prior $A r$ temia ingestion (open circles), initial responsiveness to mechanical stimulation followed by the habituation of oral disk contraction is quite evident (Trial Blocks 3-16). Figure 2 presents data from a subject in the water exchange condition in which the ingestion of Artemia had minimal effect on initial responsiveness to mechanical stimulation. Even here, however, some suppression was evident on later trials. Comparison of the data collection with and without prior Artemia ingestion reveals that substantially more responding occurred across the complete 70-trial sequence in the absence of Artemia ingestion.

Analysis of the changes occurring throughout the 70-trial sequence permits the assessment of any recovery in Anthopleura paralleling that described in

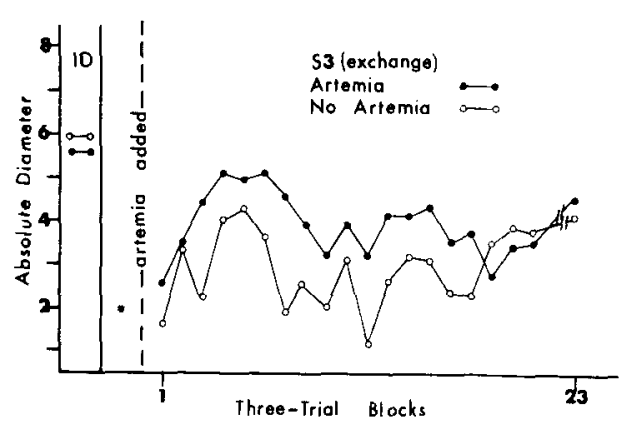

Figure 2. Three-trial block means of the subject's responding to water-stream stimulation both following and in the absence of prior ingestion of live Artemia salina. The data represented were collected under conditions of complete exchange of the fluid medium. (* = responsiveness to water-stream prior to ingestion; ID = initial resting diameter.)

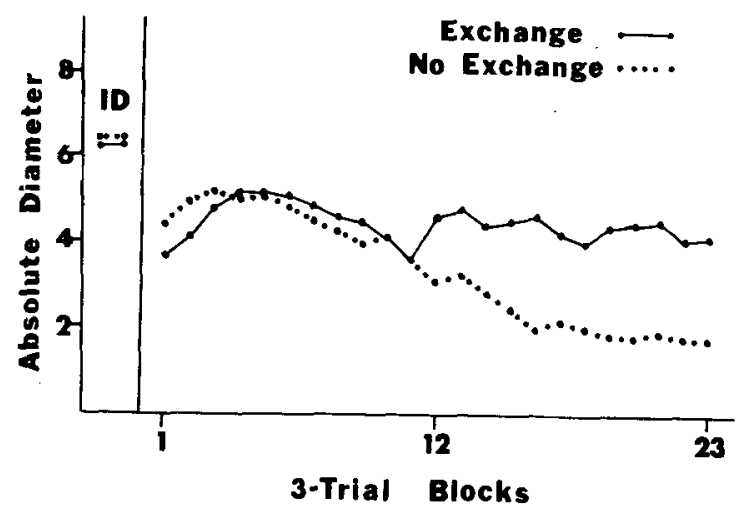

Figure 3. Three-trial block means of responding to water-stream stimulation averaged across the four subjects tested repeatedly under conditions of water exchange $(\bullet-\longrightarrow)$ and no water exchange $(\bullet \bullet \bullet)$ ). (ID = initial resting diameter averaged across the four subjects.)
Hydra (Rushforth et al., 1964). The data presented in Figure 3 describe changes in responsiveness following Artemia ingestion averaged across the four animals tested both under conditions of water exchange and no water exchange. It is evident that no substantial recovery occurred in these animals in the absence of the accumulation of fresh water in the beaker. However, in the presence of that accumulation, when no water exchange took place, apparent recovery was observed during the second half of the 70-trial test sessions. Comparison of the two conditions suggests that this recovery resulted not from a gradual decrease in the inhibitory effects of Artemia ingestion, but from the subjects' sensitivity to greater and greater proportions of fresh water in the beaker. Over the 70-trial sequence in the absence of water exchange, the volume of water in the beaker rose from a maximum of $150 \mathrm{ml}$ at the beginning of a session to as much as $350 \mathrm{ml}$ by the end of the session, a roughly $50 \%$ decrease in the salinity of the seawater. This very likely resulted in a resumption of responding during the latter half of the session. Comparison of differences in the amplitude of responding over the last 35 trials in the exchange vs. no exchange conditions yielded a $t(3)=13.24, p<.01$.

\section{DISCUSSION}

Rushforth (1973) has proposed a neurophysiological model of the Hydrozoan nervous system that attempts to distinguish among neural systems underlying the several forms of behavioral inhibition described in Hydra. Suppression of contractions of the body column follow repeated mechanical stimulation (habituation), presentation of a single photic stimulus, and the single administration of reduced glutathione or Artemia. Demonstrated interactions occur in Hydra among pacemaker systems underlying contraction of the body column, tentacle contraction, and elongation of the body column. The suppressive effects of photic stimulation are assumed to result from inhibition by the latter system on those generating contraction of the body column and tentacles. The suppressive effects of exposure to $A r$ temia and GSH, however, are not accompanied by activation of the system underlying column elongation, and these stimuli are, therefore, assumed to act directly on the systems activating contractions of the column and tentacles. Rushforth (1973) suggests that the mechanisms underlying the suppressive effects of the ingestive substances are distinct both from those producing photic suppression and from those which result in behavioral habituation. The results reported here indicate that ingestion of Artemia has a similarly suppressive effect on the magnitude of oral disk contractions in the Anthozoan Anthopleura elegantissima. These find- 
ings raise the possibility that the mechanisms of Artemia-induced inhibition in Anthopleura may parallel those hypothesized for Hydra. Pacemaker interactions are known to characterize throughconducting systems in the nerve nets of both groups, and GSH plays a central role in the ingestion response of each.

Rushforth (1967) has reported the recovery of responsiveness following ingestive inhibition after from 1 to $7 \mathrm{~h}$ in Hydra, depending on GSH concentration. The data presented in Figure 1 suggest the apparent recovery of responding in Anthopleura as well. Responding has recovered after approximately 55 trials in the condition in which no water exchange took place during testing. However, similar recovery evident in the control condition (no Artemia) strongly indicates that the result does not represent a decline in the inhibitory effects of Artemia (as in Hydra), but here results from the absence of water exchange. The data described in Figure 3, comparing "recovery" with and without water exchange, indicate that in the absence of exchange no recovery is evident in the later trials. The animal remains unresponsive to the water stream after over $1 \mathrm{~h}$ of repeated stimulation. It appears that in the former case the resumption of responding is not true recovery, but, rather, reflects the animal's sensitivity to the accumulation of fresh water in the beaker, a situation which does not obtain with the fluid exchange in effect. The failure to obtain recovery under conditions of fluid exchange may represent either a far longer time course required for recovery in these animals as compared with Hydra or an interaction between the suppressive effects of Artemia and the concurrent occurrence of habituation as a function of repeated sitmulation (Logan, 1975). That is, in the early trials, failure to respond is due to Artemiainduced suppression, while in the later trials responding may have habituated with repeated stimulation. Rushforth et al. (1964) report no interaction between habituation and Artemia-induced suppression; however, previous reports have established that habitua- tion occurs in Hydra only after several hours of mechanical stimulation.

On the basis of the results reported here, it appears that the ingestion of Artemia salina produces a suppression of responsiveness to mechanical stimulation in Anthopleura elegantissima. Similarities to comparable effects described for Hydra suggest that neurophysiological models of inhibitory processes devised for Hydrozoans (Rushforth, 1971, 1973) may more generally apply to Anthozoans as well.

\section{REFERENCES}

Jennings, H. S. Modifiability in behavior. I. Behavior of sea anemones. Journal of Experimental Zoology, 1905, 2, 447-473.

LENhoff, H. M. Behavior, hormones, and hydra. Science, 1968, 161, 434-442.

LINDSTEDT, K. J. Biphasic feeding response in a sea anemone: Control by asparagine and glutathione. Science, 1971, 173, 333-334.

LogAN, C. A. Topographic changes in responding during habituation to water-stream stimulation in sea anemones (Anthopleura elegantissima). Journal of Comparative \& Physiological Psychology, 1975, 89, 105-117.

LOGAN, C. A., \& BEck, H. P. Long-term retention habituation in the sea anemone (Anthopleua elegantissima). Journal of Comparative and Physiological Psychology, 1978, 92, 928-936.

Loomis, W. F. Glutathione control of the specific feeding reactions of Hydra. Annals of the New York Academy of Science, 1955, 209-288.

Rushforth, N. B. Chemical and physical factors affecting behavior in Hydra: Interactions among factors affecting behavior in Hydra. In W. C. Corning \& S. C. Ratner (Eds.), Chemistry of learning. New York: Plenum, 1967.

RushForTh, N. B. Behavioral and electrophysiological studies of Hydra I. An analysis of contraction pulse patterns. Biological Bulletin, 1971, 140, 255-273.

Rushforth, N. B. Behavioral modifications in coelenterates. In W. C. Corning, J. A. Dyal, \& A. O. D. Willows (Eds.), Invertebrate learning (Vol. 1). New York: Plenum, 1973.

Rushforth, N. B., Krone, I., \& Brown, L. K. Behavior in Hydra: Inhibition of the contraction responses of Hydra pirardi. Science, 1964, 146, 602-604.

(Received for publication June 22, 1978; revision accepted October 16,1978 .) 